\title{
Percutaneous Angioplasty of Coronary Obstruction in an Infant on Extracorporeal Membrane Oxygenation
}

\author{
Heather A. Sowinski, DO ${ }^{1,2}$, Bennett P. Samuel, MHA, BSN, RN', Vishal R. Kaley, MBBS, MD', \\ E. Oliver Aregullin, MD, FAAP ${ }^{1,2}$, Giedrius Baliulis, MD ${ }^{1,2}$, Neal D. Hillman, MD ${ }^{1,2}$, \\ Marcus P. Haw, MBBS, FRCS, FECTS ${ }^{1,2}$, Joseph Vettukattil, MBBS, MD, DNB, CCST, FRCPCH, FRSM, FRCP $1,2^{*}$ \\ ${ }^{1}$ Congenital Heart Center, Spectrum Health Helen DeVos Children's Hospital, Grand Rapids, Michigan, USA \\ ${ }^{2}$ College of Human Medicine, Michigan State University, Grand Rapids, Michigan, USA
}

\begin{abstract}
The arterial switch operation (ASO) is the standard surgical technique for transposition of the great arteries. Although there have been significant improvements in long-term outcomes in patients undergoing the ASO when compared to the atrial switch procedure (Mustard or Senning), early and mid-term morbidity and mortality due to coronary complications have been identified. We describe percutaneous coronary artery angioplasty in a 9-week-old infant on extracorporeal membrane oxygenation status post ASO with optimal outcomes.

Copyright @ 2019 Science International Corp.
\end{abstract}

\section{Key Words}

Arterial switch operation - Coronary artery • Percutaneous coronary angioplasty.

\section{Introduction}

Coronary artery complications may contribute to significant morbidity and mortality after the arterial switch operation (ASO). If coronary artery complications are present, revision of coronary buttons or surgical revascularization is usually performed to preserve myocardial function and avoid ischemic events. We report successful percutaneous coronary artery

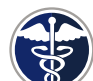

Fax +1 2037853346

E-Mail: jshd@scienceinternational.org

http://structuralheartdisease.org/ (c) 2019 Journal of Structural Heart Disease Published by Science International Corp. ISSN 2326-4004

Accessible online at: http://structuralheartdisease.org/ angioplasty with optimal long-term outcomes in an infant after ASO.

\section{Case Presentation}

A 9-week-old male infant post-surgical repair of double outlet right ventricle (DORV) and transposition of the great arteries (TGA) presented to our emergency department (ED) with cyanosis, and respiratory distress following a brief episode of poor feeding and emesis. Born at 39-weeks gestation by caesarean section at $3.12 \mathrm{~kg}$, he had prenatal diagnosis of TGA. A postnatal echocardiogram confirmed the diagnosis of DORV with TGA, inverted coronary pattern, large perimembraneous ventricular septal defect (VSD) with inlet extension, subpulmonary stenosis due to muscle bundles, and patent ductus arteriosus. He was discharged to home with stable oxygen saturations and plan for close monitoring, but readmitted at age of 3 weeks requiring balloon atrial septostomy due to significant cyanosis. Surgical repair was performed at age 5 weeks and included ASO, closure of VSD and relief of subpulmonary stenosis by resecting the right ventricular outflow tract muscle bands. His post-operative course was complicated by abdominal ascites requiring peritoneal drainage placement. He was discharged to home on post-operative day 10

* Corresponding Author:

Joseph Vettukattil, MBBS, MD, DNB, CCST, FRCPCH, FRSM, FRCP

Congenital Heart Center

Spectrum Health Helen DeVos Children's Hospital

100 Michigan NE (MC48), Grand Rapids, Michigan 49503 , USA

Tel. +1 616267 0988; Fax: +1 616267 1408;

E-Mail: joseph.vettukattil@helendevoschildrens.org 


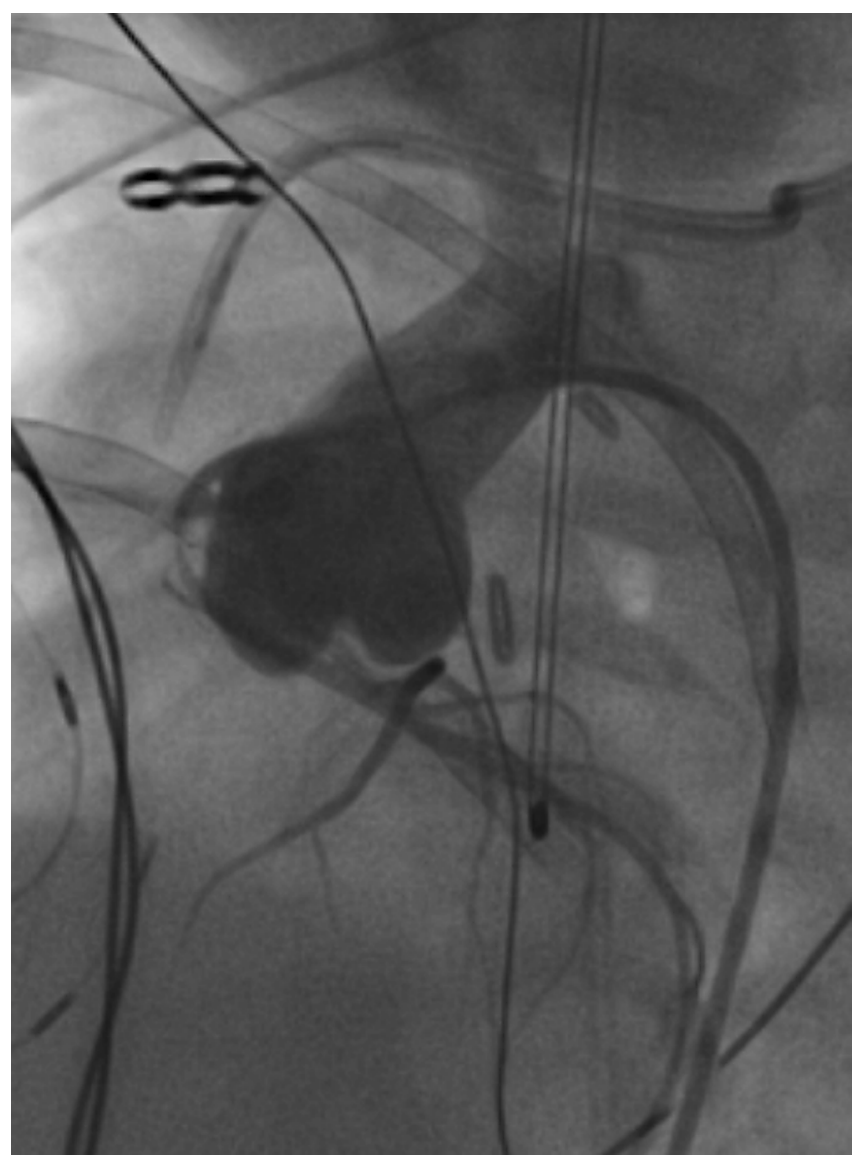

Figure 1. Initial lateral angiogram, with cranial and LAO angulation of the LMCA obtained with a non-tapered angled coronary guide catheter. The initial segment of the LMCA has a diminished filling and caliber. The proximal part of the LAD and the circumflex arteries are also seen. There is back filling of the ascending aorta and right coronary artery.

without any complications noted at his follow-up visit at age 8 weeks. The following week, he presented in respiratory distress.

Upon evaluation in the ED, the patient had supraventricular tachycardia with heart rate in the $160 \mathrm{~s}$ that reverted to normal sinus rhythm following initiation of antiarrhythmic medications (esmolol and digoxin). Electrocardiogram (ECG) showed T-wave inversion in lead $\mathrm{QVL}$ and minor ST segment elevation in leads 2, 3 and aVF. Transthoracic echocardiogram demonstrated severely impaired biventricular systolic function. Subsequently, he developed 2:1 atrioventricular block requiring intubation, temporary pacing and adjustment of antiarrhythmic medications. His cardiac enzymes were elevated with Troponin $\mathrm{T}$ at
$2.23 \mathrm{ng} / \mathrm{mL}$. A computed tomography (CT) angiogram was performed due to the unknown etiology of the poor biventricular systolic function and showed marked narrowing of the left main coronary artery (LMCA), which in this case was on the right side of the aorta, and occlusion of the left anterior descending artery (LAD).

Considering the history and $\mathrm{CT}$ angiogram findings, the patient was taken to the operating room for possible coronary revascularization. Temporary epicardial pacing was initiated due to $3: 1$ and 4:1 heart block, which led to immediate loss of cardiac output requiring placement on cardiac extra corporeal membrane oxygenation (ECMO). Significant inflammation and adhesions were observed around the LMCA. The remains of topical hemostatic agent was found surrounding the LMCA as it curved around posterior to the pulmonary artery. The LMCA was significantly different in appearance from the proximal artery, however, there was no kinking or twisting. The button was inspected and found to be in perfect position. The dense fibrotic and coagulated material around the LMCA was dissected and released, but myocardial function did not instantly improve so the patient remained on ECMO without significant change in left ventricular function.

Without any improvement of myocardial function, cardiac catheterization was performed to reassess the results of the surgical intervention and coronary blood flow on this $4.5 \mathrm{~kg}$ infant. The femoral artery was cannulated with a $4 \mathrm{Fr}$ sheath. A $4 \mathrm{Fr}$ non-tapered angled coronary guide catheter was used to perform selective coronary angiograms, which delineated the presence of persistent narrowing in the proximal LMCA segment (Figure 1). A 0.014 balance middle weight guidewire was then passed into the LMCA and carefully positioned in the LAD. A $1.5 \mathrm{~mm} \times 12$ $\mathrm{mm}$ Apex $^{\mathrm{TM}}$ PTCA dilatation catheter (Boston Scientific Corporation, Marlborough, MA) was passed over the coronary wire. Multiple inflations were performed to obliterate the waist. The wire and the balloon were removed after selective angiography of the LMCA, which showed significant improvement in flow (Figure 2).

Four days after the procedure, the patient was able to be weaned off ECMO support. Within 9 days, the ventricular systolic function returned to normal with 


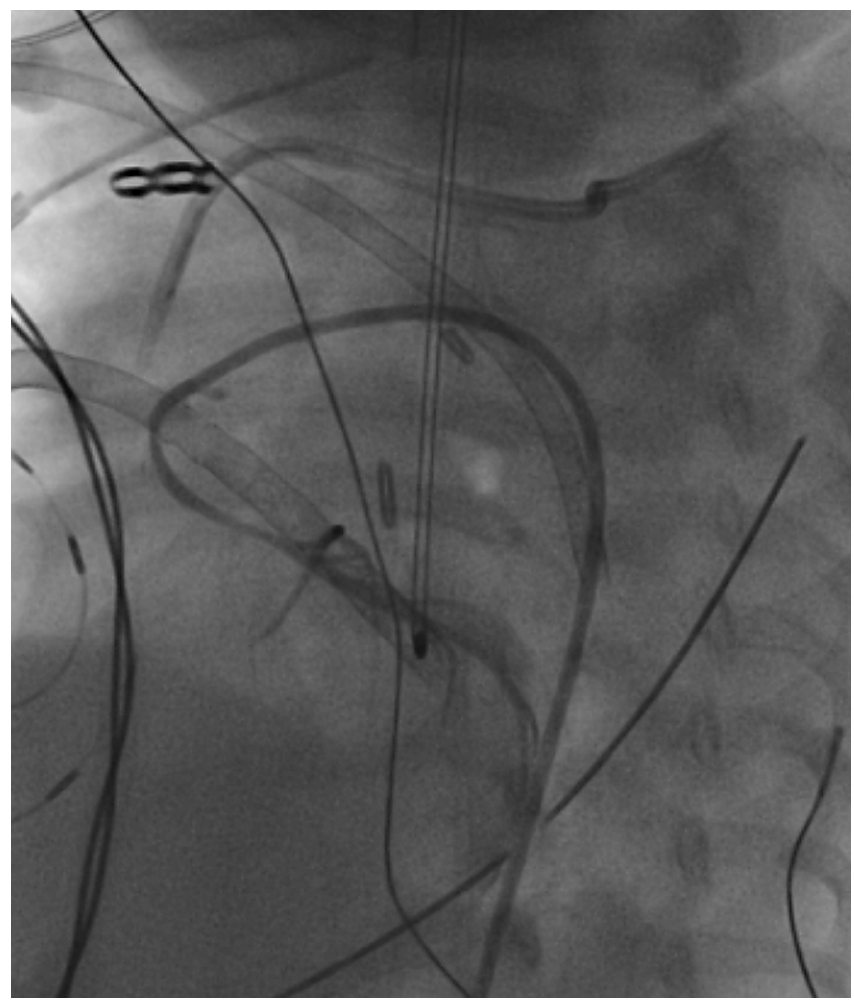

Figure 2. Lateral angiogram following percutaneous coronary angioplasty with cranial and LAO angulation of the LMCA obtained with a non-tapered angled coronary guide catheter. The initial segment of the LMCA has improved filling and caliber. The proximal part of the LAD and the circumflex arteries are also seen.

ejection fraction of $73 \%$. The patient was discharge home 1 month after the procedure. At a follow-up visit, five months after the percutaneous coronary artery angioplasty, echocardiogram showed optimal flow through the coronary arteries, normal left ventricular systolic function with ejection fraction of $59 \%$.

\section{Discussion}

The ASO has become the standard method for surgical correction of TGA since its introduction by Jatene and colleagues in 1975 [1]. Although there has been a significant decrease in morbidity and mortality rates when compared to the atrial switch procedure operation and long-term outcomes, the risk of acute and subacute coronary artery complications is well recognized after ASO [2]. Surgical revascularization of the coronary artery is usually recommended to preserve myocardial function and avoid ischemic events if coronary artery complications are observed [3]. However, consideration must be given to alternative explanations for myocardial dysfunction especially after a period of weeks of normal recovery. The use of glutaraldehyde fixed hemostats should be avoided as evidenced by the inflammation of the coronary arteries in our patient. The case suggests that percutaneous coronary artery angioplasty may be an alternative to highly surgically demanding coronary artery revascularization in a small infant. Our experience shows that coronary artery balloon dilation can be performed safely and effectively in infants with inflammatory stenosis of the coronary arteries even on ECMO. Stent angioplasty was not possible in this infant due to the extremely small size of the coronaries. The potential for coronary in-stent restenosis and need for further repeated interventions with growth was also a concern. The prompt suspicion of coronary artery complications after ASO and timely intervention can rescue the left ventricular function and result in a complete recovery with optimal long-term outcomes. Percutaneous angioplasty must be considered in infants with post-operative coronary stenosis.

\section{Conflict of Interest}

The authors have no conflict of interest relevant to this publication.

Comment on this Article or Ask a Question 


\section{References}

1. Jatene $A D$, Fontes VF, Paulista PP, Souza LC, Neger F, Galantier M, et al. Anatomic correction of transposition of the great vessels. J Thorac Cardiovasc Surg 1976;72:364370. PMID: 957754

2. Rudra HS, Mavroudis C, Backer CL, Kaushal $\mathrm{S}$, Russell H, Stewart RD, et al. The arterial switch operation: 25-year experience with 258 patients. Ann Thorac Surg 2011;92:1742-1746. DOI: 10.1016/j.athoracsur.2011.04.101
3. Agnoletti $G$, Bajolle F, Bonnet $D$, Sidi $D$, Vouhé $P$. Late coronary complications after arterial switch operation for transposition of great arteries. Clinical and therapeutic implications. Images Pediatr Cardiol 2005;7:1-11. PMID: 22368651
Cite this article as: Sowinski HA, Samuel BP, Kaley VR, Aregullin EO, Baliulis G, Hillman ND, Haw MP, Vettukattil J. Percutaneous Angioplasty of Coronary Obstruction in an Infant on Extracorporeal Membrane Oxygenation. Structural Heart Disease. 2019;5(1):710. DOI: https://doi.org/10.12945/j. jshd.2019.013.18 